\title{
Research of CAPP System Based on the Solid Edge
}

\author{
CUI Jiang-tao ${ }^{1, \text { a }}$, SHEN Guo-qiang ${ }^{1, b}$ \\ ${ }^{1}$ College of Mechanical Engineering, HANGZHOU DIANZI UNIVERCITY, Hang Zhou, China \\ a1046825238@qq.com, ${ }^{b} 19064019 @ q q . c o m$
}

Keywords: Solid Edge ; 3D-CAPP ; System Development ; Database

\begin{abstract}
The three-dimensional CAPP prototype is studied based on Solid Edge platform using the technology of secondary development of VB. The system is able to complete part the selection of machining feature of the part, edit of process information and selection of process. The system finally output process card semi-automatic or automatic, through the module of process edit and the module of process card output module having the support of the process resource management module.
\end{abstract}

\section{Introduction}

With science and technology's advancing by leaps and bounds in modern social, information and knowledge sharp growth, manufacturing enterprises are ushering in unprecedented opportunities and challenges. With the maturing of computer network information technology and three-dimensional CAD technology, three-dimensional technology is widely used in manufacturing. At the same time, CAPP technology and software industry have entered a new stage. Between CAD and CAM, CAPP has played a role of a bridge, requiring that it can extracted geometry, process away directly from the 3D model parts material and can output the tool list, cutter location file and process planning information in the follow-up process . Seen in this light, to research and develop the CAPP system and to promote and application have a far-reaching significance. It can shorten production preparation cycle and make the process more normalization and standardization ,it can improve the quality of process design , it can reduce effectively the repeatability of the process design staff labor; Finally it can reach a purpose of rapid increasing in the level of technology through the process of experience accumulation ${ }^{[1] \mathbf{I} 2]}$.

\section{Characteristics of Three-dimensional CAPP System}

With the advancement of science and technology, the CAPP system has developing ,but its own shortcomings still exist. For example, CAPP system has big dependence to more experience people and has limitations in all areas. All existing CAPP systems are difficult to achieve a good product

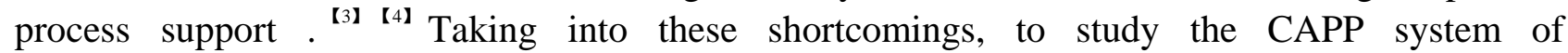
three-dimensional environment is extremely important. It can solve the above shortcomings, the features as follows:

$1>$ Feature technology in three-dimensional modeling can accurately record parts geometric features and non-geometric characteristics and record the information of the relevant elements, having parameters, fully associative and intelligent features. it is able to reflect accurately the generation, associated and modify of the relevant elements .

2>Plenty of sources of information, the 3D part models created in 3D software environment comes with a lot of attribute information of part, like the parts attribute information, process information, etc., so that you can provide sufficient information to 3D CAPP system.

$3>$ It is help to a summary of the design methods and design experience. Through 3D CAPP system make feature analysis and information extraction to part and make Classification, organize, summarize and accumulation according to the different processing methods.

$4>$ It is easy to choose the right modeling approach, saving time. Part in the three-dimensional environment modeling techniques, combined with CAPP system , can make process design work 
and greatly reduce the complexity and difficulty of the work of the CAPP system ${ }^{\mathbf{5 1} \mathbf{1} 6 \mathbf{1}}$

\section{Frame of 3D CAPP System Based on the Solid Edge}

Work Process of 3D CAPP System. First, the three-dimensional software designers built the model of three-dimensional parts and open CAPP system through the plug-in menu of the menu bar in the Solid Edge environment. Then, the user need to choose the rough shape ,processing characteristics and so on .Through the interactive interface and intelligent, the system automatic or semi-automatic choose various information, used machining features, such as: machine tools, props, fixture gage, feed rate, feed rate, and the route of walked as well as the processing parameters. After the above operation is completed, the system will automatically fill process card and can be displayed to the user, the user can printout after checking the correct.

Process of 3D CAPP System.3D CAPP system workflow (refer with : Fig. 1): First of all, the user opens the Solid Edge software, entering the part modeling plate, selecting open the part from the menu bar, select a three-dimensional parts; then open the CAPP applications from drop-down menu bar and log the user. By interactive, add parts processing requirements in the three-dimensional part model; This way, you can see intuitive processing requirements in the three-dimensional part model when you edit process; Finally, user input the process information of parts and printout process card after checking

Information Flow of 3D CAPP System.3D CAPP system information flow (refer with : Fig. 2) as follows: First, users still need to open the part model, as described above; Then, the user needs to add processing requirements to the processing of entry module , these process information will be attached to the geometric characteristics of the three-dimensional parts ,ok to save, the system will generate an XML file, This file contains processing requirements and characteristics, machined parts, in the three-dimensional model; Then, through the process edit module of 3D CAPP system, the file is read. Data information is displayed in user interface of the process of editing module, and thus as a reference when edit process.

Processing editing module requires the support of processing resources database management module, processing resources database management module make name or coded of all processing resources transfer to process editing module, allowing make the user to choose.

After finishing process edit, make a craft audit, and then , the user enters a process card output module. First, the user needs to select the card template, and then the system will fill in the content process card , which is the needed information, by this template, the user audit the errorless and output process card.

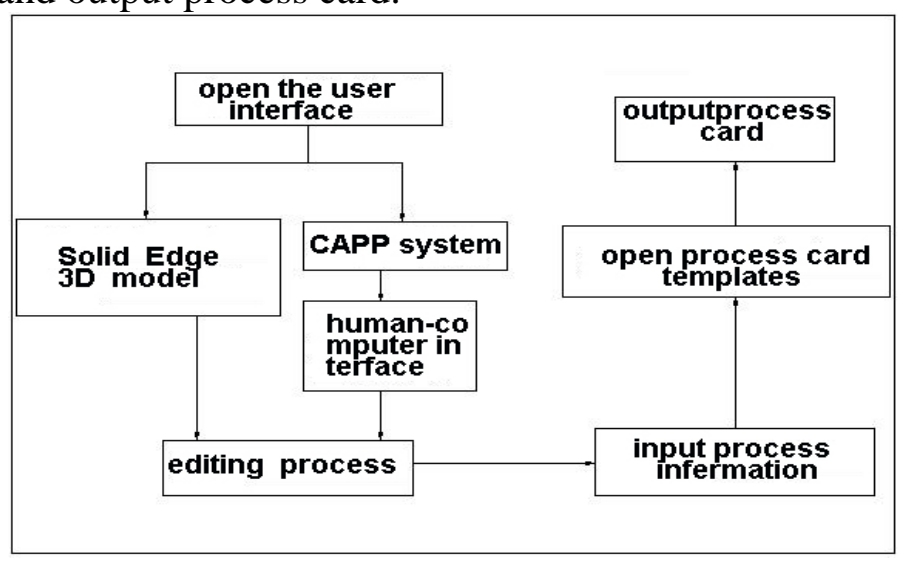

Fig. 1

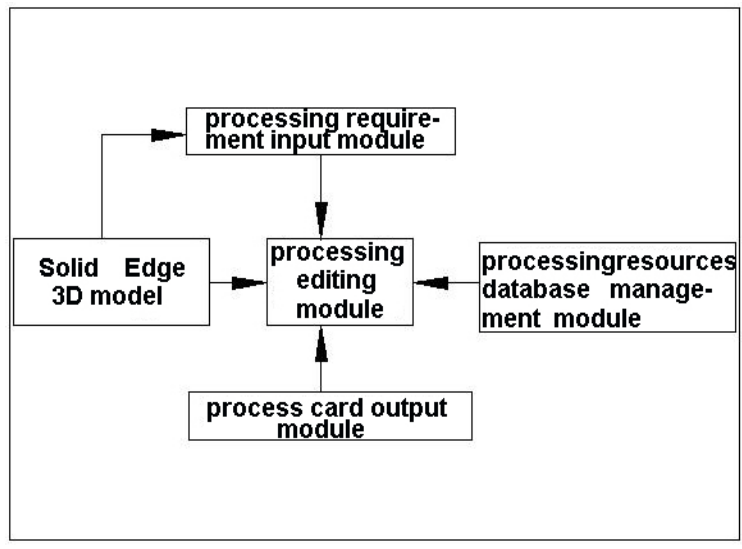

Fig. 2

\section{Overall Design of 3D CAPP System}

After making general design, you need to do an overall design of the system .The overall design ,based on the Solid Edge 3D CAPP system ,mainly includes functional module design, the database module design and the design of the user interface module. 
Features Modular Design of 3D CAPP System.As for the 3D CAPP system ,based on the Solid Edge ,it is divided three parts from the function : the editing part of the system, information and document management section and system security and maintenance section.

System Edit Part.This part is mainly edit drawings and the technical documents which related with drawings. This system can achieve the edit to solid edge files, word documents and Excel files.

Information and Document Management Section.The key core part of this paper is the part of management of information and documents. The main content of this part is part information management, pattern processing management, drawing information management. Parts information management is mainly to manage the part number, the the engineering command list, engineering command ID; the pattern processing the management major is mainly manage the code of the part to be machined, the characteristics of process, as well as the ability of the device; drawings information management is mainly to manage the product parts and components.

System Maintenance and Security Section.We should be responsible for the system's safety and its need to be maintained easily. This part is to be achieved by setting the permissions.

Database Module Design of 3D CAPP System .Good system is needed to be supported by a healthy database. By contrast a variety of database ,I find the SQLSERVER database more convenient, fast. What's more, it has a graphical user interface, and make system management and database management more intuitive .Therefore I choose SQLSERVER database.

I established a plurality of database In the SQLSERVER database (as shown in Fig. 3, Fig. 4), using to store the user's various information.

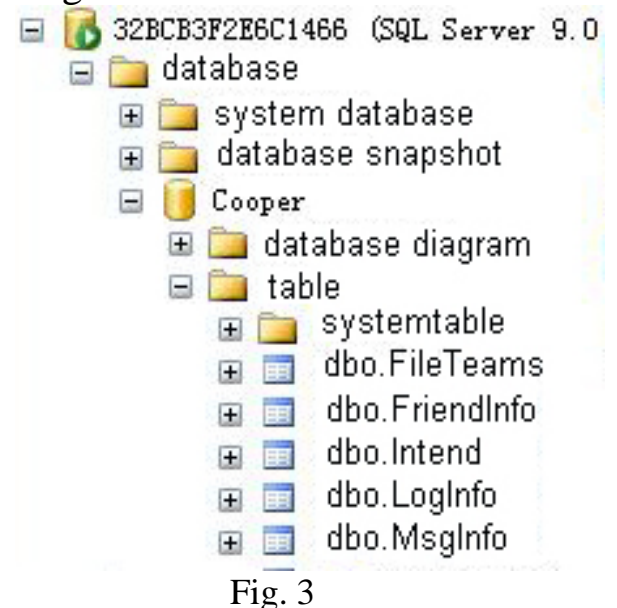

Fig. 3

\begin{tabular}{|c|c|c|c|c|c|c|c|}
\hline \multicolumn{3}{|c|}{ table dbo.FileTeans } & \multicolumn{5}{|c|}{ summary } \\
\hline & FileID & ProjectID & FileName & FilePath & Filesize & Filestate & FileDescription \\
\hline & 946 & 212 & 2cping $2 \ldots$ & animal |avred & MUI & 1 & MUI \\
\hline & 947 & 212 & 2cping2... & human & MUI & 0 & MUI \\
\hline & 948 & 212 & guidao $2 \ldots$ & plant'lavred & Mul & 1 & MUI \\
\hline \multirow[t]{6}{*}{ 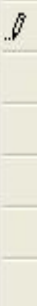 } & 949 & 212 & guida02... & fumiture & nul & 2 & null \\
\hline & 968 & 217 & good? Es... & kkiavred & MUI & 0 & nur \\
\hline & 969 & 217 & goodes... & kk & Moll & 0 & MUI \\
\hline & 983 & 215 & mmm20... & drivewaylhuila... & nul & 0 & MUI \\
\hline & 1018 & 211 & bbbb20... & driveway'mark & MNI & 0 & noll \\
\hline & 1068 & 215 & HMMAPI... & driveway'huil,a... & Mul & 0 & MUI \\
\hline
\end{tabular}

Fig. 4

\section{System Prototype run}

Solid Edge ST2 version of the software, click on the drop-down menu .open the CAPP system, click process files editing interface and complete process documents by way of human-computer interaction. After selecting parts click to view work step-by-step, open the parts interface of work step-by-step. Choose a three-dimensional attempt to fill in the information. Fig.5 - Fig.6 are the running instance of the part.

\section{Summary}

This article is based on Solid Edge three-dimensional modeling software, using secondary development of VB, and finally developing a three-dimensional CAPP system. It make a integration of system design and manufacturing processes, and also pave the way for the Information technology of manufacturing sector. The system is largely saving the R \& D time of part products and improving the efficiency of the overall design and making great economic benefits for the development of enterprises, it is worthy of further research and development. 


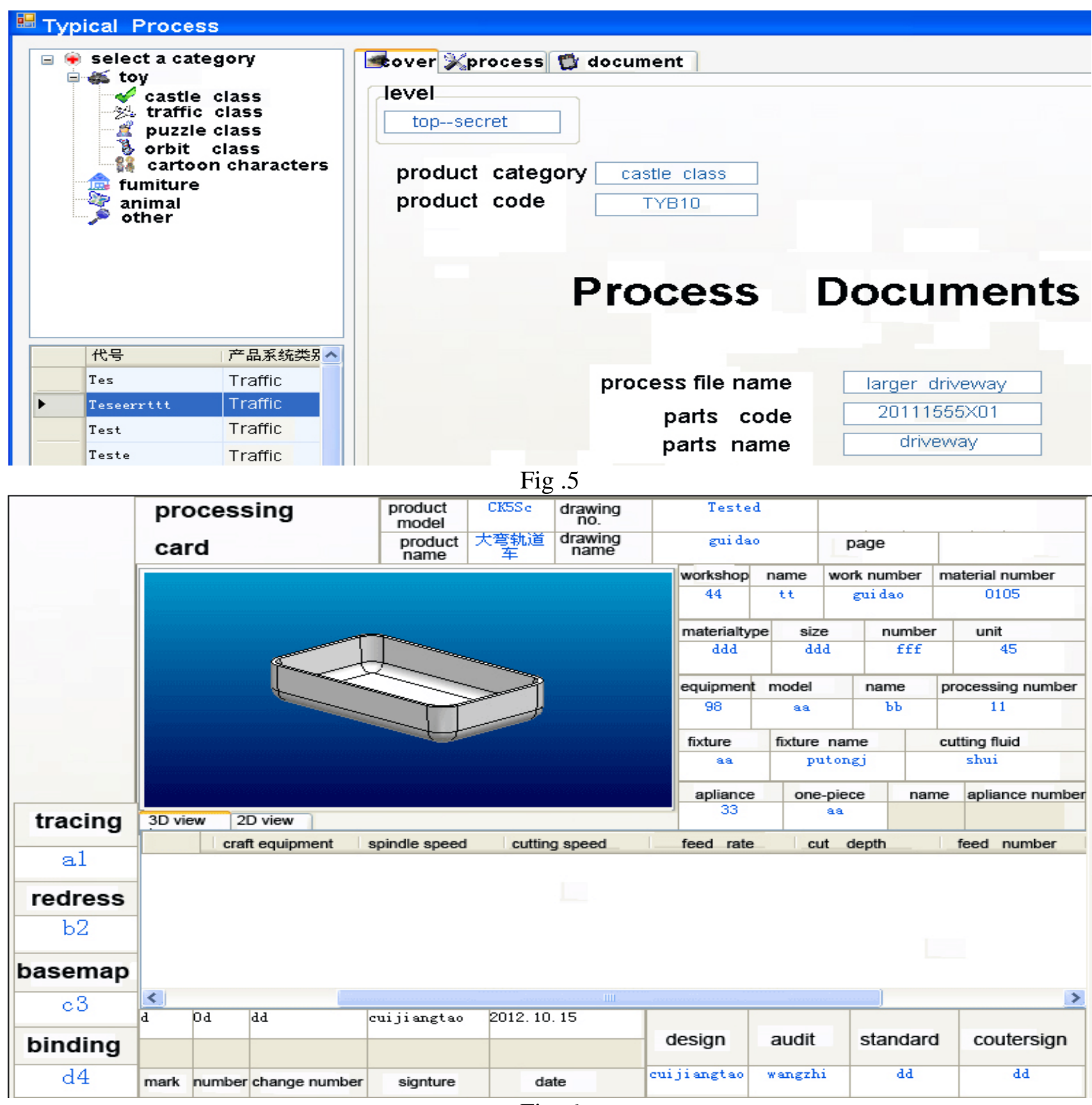

Fig. 6

\section{References}

[1]Buyun Sheng.Study and Practice on CAPP Software Component Base on 3D Platform.Machinery.2007,(4):20-22.

[2] Xinxin Liu . CAPP System Process Management Research. Modern Manufacturing Technology and Equipment.2007,(5):58-59.

[3]Li Sun. Research on the Key Techniques of CAPP Database. Machine Building \&Automation.2007,36(5):23-25

[4] Zheng Xu. Study and Practice of CAD/CAPP integrated method in 3D Environment. Machinery Design \& Manufacture.2005,(8):55-57.

[5] Zongshun Chen.machinery manufacturing process design and CAPP technology [M] Beijing: Tsinghua University Press, 2004.

[6] Rujia Zhao. aided process planning (CAPP) [M]. Beijing: Machinery Industry Press, 2003 